\title{
Proteomic analysis of human epithelial ovarian cancer xenografts in immunodeficient mice exposed to chronic psychological stress
}

\author{
GAO Jun ${ }^{1}$, GAO GuoLan ${ }^{2 *}$, ZHANG YanYu ${ }^{1} \&$ WANG Fen ${ }^{1}$ \\ ${ }^{1}$ Department of Gynecology and Obstetrics, the First Affiliated Hospital of Nanchang University, Nanchang 330006, China; \\ ${ }^{2}$ Department of Gynecology, Aviation General Hospital, Beijing 100012, China
}

Received May 9, 2010; accepted October 13, 2010

\begin{abstract}
This work aims at comparing alterations in the proteomes of human epithelial ovarian cancer xenografts between stressed and non-stressed immunodeficient mice as well as exploring the molecular mechanisms linking chronic psychological stress to ovarian cancer oncogenesis and progression. SK-OV-3 cells were injected subcutaneously into nude mice. The stress group was subjected to a chronic physical restraint protocol for $6 \mathrm{~h}$ on 35 consecutive days, while the control group was unrestrained. All mice were sacrificed on day 36 after SK-OV-3 cell injection, and tumors were excised. Tumor tissues were processed for 2D gel electrophoresis, mass spectrometry (nanoUPLC-ESI-MS/MS) and Western blotting. The expression of 20 proteins was found to be significantly altered between the stress and control groups, of which 14 were up-regulated, five were down-regulated, and one protein was found only in the stress group. All proteins were identified by UPLC-ESI-MS/MS, and Western blotting results were consistent with those of proteomic methods. The present results provide new evidence relating to the molecular mechanism underlying the relationship between psychological stress and tumor progression.
\end{abstract}

proteomic, psychological stress, ovarian cancer, 2D electrophoresis, UPLC-ESI-MS/MS

Citation: Gao J, Gao G L, Zhang Y Y, et al. Proteomic analysis of human epithelial ovarian cancer xenografts in immunodeficient mice exposed to chronic psychological stress. Sci China Life Sci, 2011, 54: 112-120, doi: 10.1007/s11427-010-4126-x

With the development of integrated treatment, remarkable achievements have been made in combating cancer. However, ovarian cancer, as the second most common malignancy of the reproductive system in women [1], remains extremely difficult to diagnose in its early stages. Most women with ovarian cancer have no, or only non-specific, symptoms. Upon diagnosis, approximately $70 \%$ of patients are already at the advanced stage. Therefore, ovarian cancer is still the leading cause of mortality among gynecological cancers, with the five-year survival rate being only 19\%-39\% [2]. Because patients suffer both mental and physical stress, they may find the rigors of therapy very daunting. This additional stress may seriously affect the prognosis.

*Corresponding author (email: guolan_gao.1962@yahoo.com.cn)
Significant advances in medicine have led from the single biomedical model of treatment to a more integrated biopsychosocial model. Factors such as socio-economic status, lifestyle, personality, and emotional and psychological factors are now taken into consideration in addition to the pathological, biological, genetic and biochemical etiology of the disorder. Clinicians and researchers alike now pay close attention to the effects of psychosocial factors on tumor patients. Studies suggest that psychosocial factors affect the immune system primarily through dysfunction of the hypothalamus-pituitary-adrenal axis. Thus, these factors affect the occurrence and prognosis of cancer [3,4]. However, most of the clinical and experimental research to date is limited to the effects on neuroendocrine and immune function. The molecular mechanisms underlying these effects are rarely defined, and there are, to our knowledge, no stud- 
ies that have reported differences in tumor protein in response to psychosocial disturbances.

Proteomic analysis, providing global protein information, is of great virtue for oncological studies. Tumor-associated proteomics is feasible and practicable to the investigation of oncogenesis, screening of cancer biomarkers, early diagnosis and treatment of cancer and assessment of prognosis [5]. Proteomic analysis allows comparisons to be made between protein expression patterns in different tissues/cells or fluctuations in protein expression elicited by different pharmaceutical or psychological treatments. These proteins can subsequently be used as potential biomarkers for monitoring studies, and help in drawing conclusions regarding the molecular mechanisms by which certain diseases arise and progress. Recently, some progress has been made using comparative proteomic studies to investigate ovarian cancer [2,6-13]. Several studies have reported hippocampus proteome changes after chronic psychological stress [14-16]. However, little is known about the effects of chronic psychological stress on the proteome of ovarian cancer tissue.

Using comparative proteomics methods, we have established a subcutaneous xenotransplanted tumor model of human epithelial ovarian cancer in nude mice and created a restraint model of chronic psychological stress. We then used high resolution two-dimensional electrophoresis (2-DE) to delineate the patterns of protein expression in the graft tumor. Proteins that were differentially expressed between stress and non-stress ovarian cancer tissue were identified by ultra-high-performance liquid chromatography-electrospray ionization tandem mass spectrometry (UPLC-ESI-MS/MS). Our aim is to investigate the molecular mechanism by which chronic psychological stress affects ovarian cancer oncogenesis and development, which will hopefully lead to better psychological and molecular treatment strategies.

\section{Materials and methods}

\subsection{Chemicals}

RPMI 1640 and fetal bovine serum (FBS) were obtained from Hyclone Corporation (USA). Sodium dodecyl sulfate (SDS), dithiothreitol (DTT), Tris (Tris-(hydroxymethyl)aminomethane), bromophenol blue, ammonium persulfate (APS), glycine, acrylamide, Immobiline Dry-Strips (3-10 L), immobilized pH gradient (IPG) buffer, Drystrip cover fluid, agarose, urea, Coomassie brilliant blue G-250, and 2-D SDS-PAGE standards were purchased from Amersham Pharmacia Biotech AB (Uppsala, Sweden). Dimethyl sulfoxide (DMSO), bovine serum albumin (BSA) trifluoroacetic acid (TFA), N,N'-methylene-bis-acrylamide, N,N,N',N'tetramethylethylenediamine (TEMED), Iodoacetamide (IAA), 3-[3-(cholamidopropyl) dimethylammonio]-1-propanesulfonate (CHAPS), protease inhibitor cocktail, and trypsin were purchased from Sigma Corporation (USA). DNase,
RNase, anhydrous sodium acetate, anhydrous sodium carbonate, silver nitrate, thiosulfate sodium, glutaraldehyde, ethanol, glacial acetic acid, methanol, acetonitrile (ACN), glycerol, formaldehyde, and Tween 20 (analytical grade or chromatography pure) were all obtained domestically. All buffers were prepared with Milli-Q purified water (Millipore, USA).

\subsection{Instruments}

The UV-160 spectrophotometer was purchased from Shimadzu Corporation (Japan). The IPGphor isoelectric focusing system, image scanner, constant temperature circulator, image analysis software (ImageMaster 2D Platinum ${ }^{\mathrm{TM}}$ ) were purchased from Amersham Pharmacia Biotech AB (Sweden). The Protean IIxi ${ }^{\mathrm{TM}}$ cell SDS-PAGE electrophoresis system was purchased from BIO-RAD Ltd. (USA). The nanoUPLCESI-MS/MS system was purchased from Waters (USA).

\subsection{Cell line and animals}

The human ovarian cancer cell line, SK-OV-3, was obtained from the Institute of Biochemistry and Cell Biology, Shanghai Institute of Biological Sciences, Chinese Academy of Sciences, China in March 2009. DNA fingerprinting using short tandem repeats was applied as a characterization method, as previously described [17]. Cells were maintained in RPMI-1640 medium supplemented with $10 \%$ heat-inactivated fetal bovine serum and penicillin-streptomycin $\left(50 \mathrm{U} \mathrm{mL}^{-1}\right)$ at $37^{\circ} \mathrm{C}$ in a humidified atmosphere of $5 \% \mathrm{CO}_{2}$ and $95 \%$ air, as previously described [18]. Cells in the exponential growth phase were harvested with trypsinization, washed in Hank's solution and ice-cold PBS, and counted. After resuspension at $2 \times 10^{7}$ cells $\mathrm{mL}^{-1}$ in serum-free RPMI-1640, $100 \mu \mathrm{L}\left(2 \times 10^{6}\right.$ cells $)$ was injected subcutaneously into mice. Female BALB/c-nu mice (4-6 weeks old) were purchased from the Laboratory Animal Research Center of Hubei Province (China), and housed in laminar air flow cabinets under pathogen-free conditions with a 12-h light/12-h dark schedule and fed autoclaved standard chow and water ad libitum [19]. All experiments on mice were conducted in accordance with the guidelines of the National Institutes of Health for the Care and Use of Laboratory Animals. The study protocol was also approved by the Animal Ethics Committee of the Medical College of Nanchang University.

\subsection{Tumor xenograft and chronic restraint stress}

Mice were divided randomly into stress and control groups after a week in the new environment (six mice per group). For the chronic restraint stress group, mice were subjected to an established chronic physical restraint protocol [20,21], in which they were restrained daily for $6 \mathrm{~h}$ (from 11 a.m. to 5 p.m.) in a $50 \mathrm{~mL}$ conical centrifuge tube filled with multi- 
ple punctures to allow ventilation, food and water, which were provided only during the stress interval. The mice were neither physically compressed nor experiencing pain. SK-OV-3 was injected subcutaneously into both stress and control group mice on the right lateral chest wall in close proximity to the axilla [22] on day 8 after starting the stress protocol. Stress group mice were exposed to stress for $6 \mathrm{~h}$ per day for 35 consecutive days after tumor xenograft, while control littermates were kept in their original cage. All mice from each group were sacrificed by cervical dislocation on day 36 after SK-OV-3 injection, and tumors were removed. The entire tumor mass was carefully dissected (excluding any extraneous host tissue), cleaned of clots, immersed in liquid nitrogen immediately after resection and stored at $-80^{\circ} \mathrm{C}$ until analysis.

\subsection{Protein extraction}

Frozen xenografts tissue samples were ground in liquid nitrogen, and homogenized on ice by using a glass tissue grinder. For every $100 \mathrm{mg}$ tissue, $1 \mathrm{~mL}$ lysis buffer (consisting of $40 \mathrm{mmol} \mathrm{L}^{-1}$ Tris buffer ( $\mathrm{pH} \mathrm{7.5),} 7 \mathrm{~mol} \mathrm{~L}^{-1}$ urea, $2 \mathrm{~mol} \mathrm{~L}^{-1}$ thiourea, $1 \%$ DTT, $4 \%$ CHAPS, and $1 \mathrm{mmol} \mathrm{L}^{-1}$ EDTA) and $10 \mu \mathrm{L}$ protease inhibitor cocktail were added. The homogenates were sonicated (10 bursts of $5 \mathrm{~s}$, separated by $15 \mathrm{~s}$ intervals) on ice using a Digital Sonifier. After sonication, $5 \mu \mathrm{L}$ of $10 \mu \mathrm{g} \mu \mathrm{L}^{-1}$ DNase and $5 \mu \mathrm{L} 10 \mu \mathrm{g} \mu \mathrm{L}^{-1}$ RNase were added. Subsequently, the sample was incubated for $20 \mathrm{~min}$ on ice. Cellular debris was removed by centrifugation at $14000 \mathrm{r} \mathrm{min}^{-1}$ for $20 \mathrm{~min}$ at $4{ }^{\circ} \mathrm{C}$, and the supernatants were collected. The protein concentrations were quantified by Bradford protein concentration assay. Global protein samples were sub-packaged, labeled and stored at $-80^{\circ} \mathrm{C}$ until 2-DE analysis.

\subsection{Two-dimensional electrophoresis}

Protein samples $(100 \mu \mathrm{g})$ were mixed with rehydration solution $\left(8 \mathrm{~mol} \mathrm{~L}^{-1}\right.$ urea, 2\% CHAPS, $0.5 \%$ IPG buffer, 18 mmol L ${ }^{-1}$ DTT and a trace of bromophenol blue) to a volume of $350 \mu \mathrm{L}$. This sample was loaded into strip holders together with $18 \mathrm{~cm}$ Immobiline DryStrips (linear $\mathrm{pH}$ gradient from $\mathrm{pH} 3$ to 10), and the loaded strips were covered with Drystrip Cover fluid. The rehydration holders were placed in the IPGphor IEF system for passive rehydration for $12 \mathrm{~h}$ at $30 \mathrm{~V}$ at $20^{\circ} \mathrm{C}$. Subsequently, isoelectric focusing on the first dimension was carried out. The proteins were focused at $20^{\circ} \mathrm{C}$ for $1 \mathrm{~h}$ at $500 \mathrm{~V}$, then $1 \mathrm{~h}$ at $1000 \mathrm{~V}$, and finally $6 \mathrm{~h}$ at $8000 \mathrm{~V}$. After completion of the IEF program, the strips were equilibrated at room temperature in two steps: $15 \mathrm{~min}$ in an IPG equilibration buffer $\left(50 \mathrm{mmol} \mathrm{L}^{-1}\right.$ Tris-HCl solution ( $\mathrm{pH} 8.8$ ), $6 \mathrm{~mol} \mathrm{~L}^{-1}$ urea, $30 \%$ glycerol, $2 \%$ SDS, and a trace of bromophenol blue) plus $1 \%$ DTT, followed by $15 \mathrm{~min}$ in IPG equilibration buffer plus $2.5 \%$ IAA. For the second dimension, SDS-PAGE with a $13 \%$ polyacrylamide gel was used. The IPG strips were placed on the top of gel and the proteins were then separated according to their molecular weights. Electrophoresis was carried out at $20^{\circ} \mathrm{C}, 15 \mathrm{~mA} / \mathrm{gel}$ for $15 \mathrm{~min}$, followed by a $6 \mathrm{~h}$ run at $30 \mathrm{~mA} / \mathrm{gel}$ until the bromophenol blue indicator front reached the bottom of the gels. Three 2-DE gels were performed for each group.

\subsection{Gel scanning and image analysis}

After silver staining, the 2-D gels were imaged on an Image Scanner in a transmission mode, and quantitative analyses of the digitized images were performed using the Image Master 2D Platinum ${ }^{\mathrm{TM}}$ software according to the protocols provided by the manufacturer, which included background subtraction, spot detection, defining landmark annotations, and matching. The intensity of each spot was normalized to the total valid spot intensity. Gels from the control and stress group were analyzed simultaneously. Each sample was analyzed based on triplicate gels produced in identical conditions to demonstrate reproducibility and diminish experimental errors. An average gel would be selected as a standard gel in each group by matching analysis, with more homogeneous spots, fewer impurities and better representation of the spot distribution. Using image analysis software, target spots were compared to the average gel to detect increases/decreases in expression (to more than double/half that of control, respectively), and to catalogue significant inter-group variations.

\subsection{Protein identification by nanoUPLC-ESI-MS/MS}

Considering its compatibility to nanoUPLC-ESI-MS/MS analysis, we chose Coomassie Brilliant Blue G-250 staining in this study. We selected the preparative gels to perform Coomassie Brilliant Blue G-250 staining. Differentially expressed protein spots were selected, which were chosen according to the spots of silver staining gels. Twenty target spots were carefully excised from gels using a biopsy scalpel, and spot pieces were digested with trypsin in a $1.5 \mathrm{~mL}$ siliconized Eppendorf tube. Spot pieces were washed twice with Milli-Q water, destained in 50\% ACN containing 100 mmol L ${ }^{-1}$ ammonium bicarbonate for $20 \mathrm{~min}$ at room temperature, dehydrated and dried using a vacuum centrifuge. The dried gel pieces were incubated in $50 \mathrm{mmol} \mathrm{L}^{-1}$ ammonium bicarbonate containing $0.1 \mu \mathrm{g} \mu \mathrm{L}^{-1}$ modified trypsin for digestion at $37^{\circ} \mathrm{C}$ overnight $(12-16 \mathrm{~h})$. The resulting harvested peptide mixture was prepared into a sample solution as described previously [23]. Sample solution (5 $\mu \mathrm{L})$ was injected into a nano-Acquity system and subjected to nanoUPLC-ESI-MS/MS analysis. The UPLC-ESI-MS/MS system consists of a nano-ACQUITY UPLC system and a Synapt high definition mass spectrometer using an electrospray ionization source Z-spray. The ACQUITY UPLC analytical column uses a $75 \mu \mathrm{m} \times 250 \mathrm{~mm}$ BEH C18 column 
packed with $1.7 \mu \mathrm{m}$ particles, and the enrichment column is a $180 \mu \mathrm{m} \times 20 \mathrm{~mm}$ symmetry $\mathrm{C} 18$ packed with $5 \mu \mathrm{m}$ particles. The column temperature was maintained at $35^{\circ} \mathrm{C}$. Optimum separation was achieved with a gradient mobile

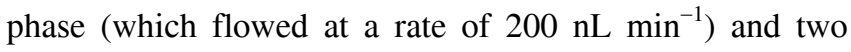
mobile phases consisting of $0.1 \%$ formic acid in water and $0.1 \%$ formic acid in ACN. The gradient conditions are 80 $\min 1 \%-40 \% \mathrm{~B}, 10 \min 40 \%-80 \% \mathrm{~B}, 10 \mathrm{~min} 80 \% \mathrm{~B}$, and $20 \mathrm{~min} 100 \% \mathrm{~B}$, then return to initial conditions. For ESI-MS/MS, ionization was achieved by using nano-electrospray ionization positive ions with a capillary voltage of $2.5 \mathrm{kV}$ and a cone voltage of $35 \mathrm{~V}$, the source temperature and desolvation temperature being set at 90 and $300^{\circ} \mathrm{C}$, respectively. Nitrogen was used as the cone gas and for desolvation, with a flow rate of 50 and $500 \mathrm{~L} \mathrm{~h}^{-1}$, respectively. Argon was used as the collision gas set at $2.5 \times 10^{-3}$ mbar. Data were acquired in data dependent acquisition (DDA) mode, and the two highest intensity ions were selected from each scan, which was carried out using tandem mass spectrometry. MS/MS spectra were processed using total data acquisition software (PLGS, v2.3), and then analyzed with the Mascot search engine (www.matrixscience.com) against the NCBInr database including two variable modifications: Carbamidomethyl (C) and Oxidation (M). One missed cleavage site was allowed for trypsin digestion, all mass values were considered monoisotopic, and the MS/MS tolerance was set at $\pm 0.2 \mathrm{Da}$. Individual ion scores of $>38$ indicate identity or extensive homology $(P<0.05)$.

\subsection{Western blotting analysis}

For Western blotting, the method was essentially as previously described [24]. Briefly, protein extracts were separated on SDS-PAGE and then blotted to polyvinylidene difluoride (PVDF) membranes (Millipore, USA). After incubating for $1 \mathrm{~h}$ with PBS containing 0.1\% Tween 20 and $5 \%$ non-fat dried milk, primary antibodies were added and the membrane was incubated at room temperature overnight. Primary antibodies in this study are rabbit anti-peroxiredoxin 1 (Millipore, USA), rabbit anti-Cu/Zn superoxide dismutase (Alexis-Bio, USA), rabbit anti-nm23 (Millipore, USA), rabbit anti-creatine kinase MM (ABCAM, UK) and glutathione-S-transferase omega 1 mouse polyclonal antibody (ABCAM, UK). After washing, goat anti-rabbit and goat anti-mouse horseradish peroxidase-conjugated secondary antibody (ZSGB, China) were added, depending on the species of the primary antibody. After incubation for $1 \mathrm{~h}$, membranes were decolorized with PBS at room temperature, subjected to enhanced chemiluminescence, and exposed to film. The exposed films were examined visually and photographed or scanned.

\subsection{Statistical methods}

To test differences in mouse body weight, xenograft weight and fold-change of protein expression between the stress and control group, $t$-tests were used (Statistical Package For SSPS17.0). $P<0.05$ was regarded as statistically significant.

\section{Results}

\subsection{Mouse body weight and xenograft weight}

Mouse body weight in the stress group was significantly lower than that in the control group after the experiment $((21.17 \pm 2.02)$ g vs. $(24.52 \pm 0.67) \mathrm{g}$, respectively; $n=6 ; P<$ $0.01)$, whereas there was no difference in body weight between the stress and control groups prior to the experiment $((18.58 \pm 1.07) \mathrm{g}$ vs. $(18.42 \pm 0.86) \mathrm{g}$, respectively; $n=6 ; P>$ $0.05)$. The subcutaneous nodules began to emerge $4 \mathrm{~d}$ after SK-OV-3 injection, and xenografts began to grow rapidly $10 \mathrm{~d}$ after SK-OV-3 injection. Average xenograft weight in the stress group was significantly higher than that in the control group $((1.72 \pm 0.57) \mathrm{g}$ vs. $(1.08 \pm 0.29) \mathrm{g} ; P<0.05)$.

\subsection{Overview of protein profiles of xenograft}

Three mice were randomly selected from each of the stress and control groups, and a single 2-D gel was prepared from each mouse. On average, 1467 protein spots were detected in stress group 2-DE profiles compared to 1292 in the control group (Figure 1). We found that matching ratios reached $92.1 \%$ (control-), $89.4 \%$ (stress-) and $82.4 \%$ (inter-group), which indicated ideal reproducibility of these images ( $>75 \%$ being considered as good). With the image analysis software, we found that 19 protein spots were expressed at significantly different levels in the stress group to the respective spots from the control group (14 were up-regulated, five were down-regulated) and one protein spot was found only in the stress group (Figures 2-4).

\subsection{Results of protein identification}

Twenty target protein spots were submitted for identification using UPLC-ESI-MS/MS analysis and NCBInr database searches. All were successfully identified. Fourteen proteins were up-regulated, including peroxiredoxin 1, laminin-binding protein, 14-3-3 protein epsilon isoform, $\mathrm{nm} 23$ protein, and phosphoglycerate kinase 1 . Five proteins were down-regulated, including $\mathrm{N}$-myc downstream regulated 1, muscle creatine kinase and myosin light chain 2 . The protein found only in the stress group gels has been identified as SET protein. Full results of the UPLC-ESIMS/MS analysis are listed in Table 1.

\subsection{Western blotting analysis}

The results showed that Prdx1, SOD1, GSTO1 and Nm23 protein expression in the stress group xenograft tissue was 

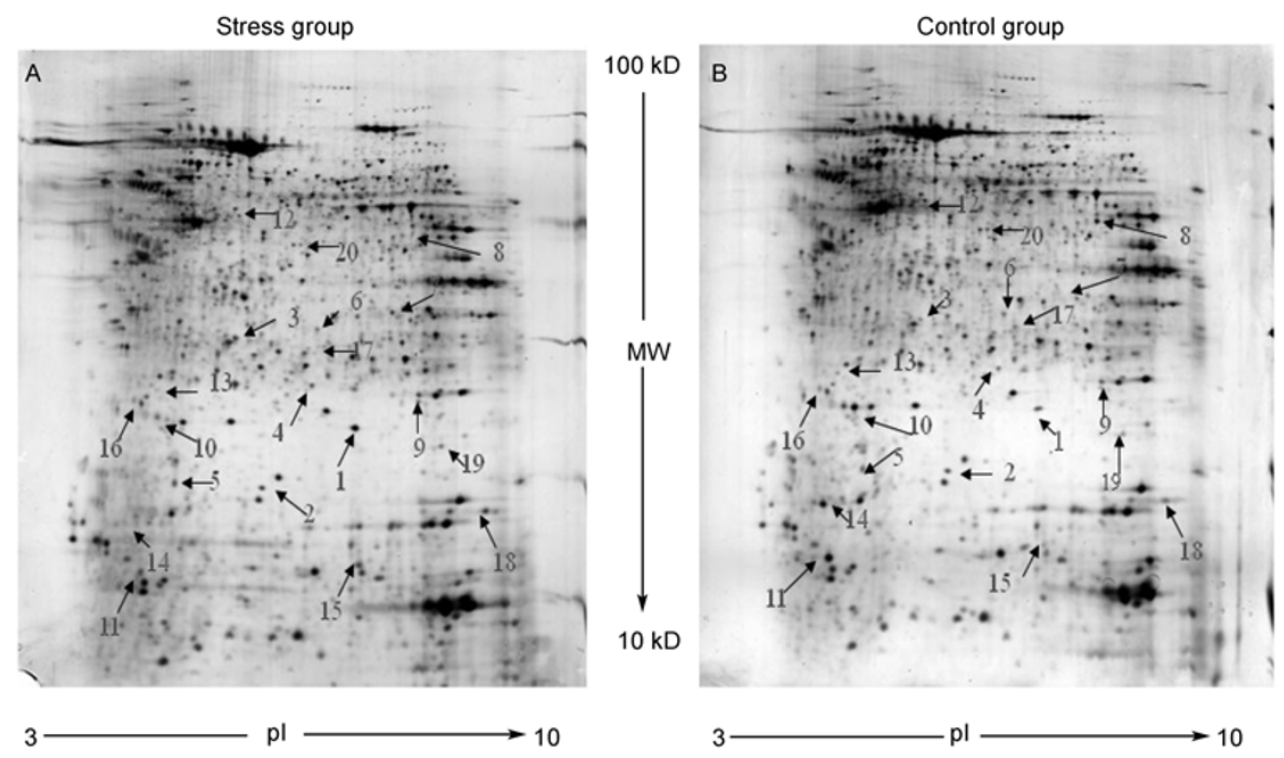

Figure 1 Representative 2-DE gels of human epithelial ovarian cancer xenograft proteins from immunodeficient mice in the psychological stress group (A) and the control group (B). Whole xenograft protein was separated by 2-DE and visualized by silver staining. Protein spots that were altered by psychological stress are labeled by numbers. The molecular weights $(\mathrm{MW})$ and $\mathrm{pI}$ scales are indicated.

higher than that in the control group, while creatine kinase MM protein expression level was significantly reduced (Figure 5), in agreement with the results of the proteomic analyses.

\section{Discussion}

Psychological factors have been shown to influence the oncogenesis and prognosis of ovarian cancer. Development and progression of ovarian cancer is a complex process involving multiple genes and signaling pathways. However, its mechanism of action remains unclear because a comprehensive study of the relationship between psychology and tumor development, and the prevention of tumor occurrence/growth by means of psychological factors, has yet to be performed. Because psychological factors are hard to control, we chose to study the effect of psychological stress on tumor development using animal models of chronic stress, which have better reproducibility/controllability, a short observation cycle and clearer results. The present study details a proteomic analysis of molecular changes in human epithelial ovarian cancer xenografts in nude mice exposed to chronic psychological stress. The study may have revealed novel proteins and signaling pathways involved in psychological stress and tumor development.

In this study, immunodeficient mice bearing human epithelial ovarian cancer cells were exposed to chronic restraint stress for $6 \mathrm{~h} \mathrm{~d}^{-1}$ for 35 consecutive days. Global protein extract was obtained from xenograft and analyzed by 2-DE and nanoUPLC-ESI-MS/MS. Twenty proteins were found to have been altered significantly between the stress and control groups. Most of them appeared to be in- volved in physiological processes, such as energy metabolism, cell proliferation, invasion and apoptosis.

Prdx1 and Prdx3 belong to the superfamily of anti-oxidant proteins, which reduce hydrogen peroxide and alkyl hydroperoxides. They may play an antioxidant protective role in cells, and contribute to the antiviral activity of CD8(+) T-cells. This protein may have a proliferative effect and play a role in cancer development and progression. The previous research has shown that a low level of $\mathrm{H}_{2} \mathrm{O}_{2}$ produced by the mitochondria regulates cell proliferation, whereas a high level of $\mathrm{H}_{2} \mathrm{O}_{2}$ was toxic to the cell and caused apoptosis. However, Prdx3-transfected prostate cancer cells show a marked resistance to hypoxia-induced $\mathrm{H}_{2} \mathrm{O}_{2}$ formation and apoptosis [25], suggesting that Prdx3 overexpression protects cancer cells against apoptosis caused by $\mathrm{H}_{2} \mathrm{O}_{2}$. Elevation of Prdx1 has been shown in oral, pancreatic, follicular thyroid, esophageal, lung, mesothelioma, breast, and head and neck cancers [26]. Moreover, up-regulation of $\operatorname{Prdx} 1$ is implicated in radiotherapy resistance of lung cancer cells and chemotherapy resistance of breast cancer, while down-regulation of Prdx 1 has been shown to sensitize lung cancer cells to radiation [26]. These studies suggest that Prdx1 enhances the aggressive survival phenotype of cancer cells, and confers an increased resistance to treatment [26]. Up-regulation of Prdx1 and Prdx3 in the stress group of mice may lead to a disadvantageous enhancement of anti-oxidant and anti-apoptosis effects in tumor cells.

Superoxide dismutases (SODs) are responsible for the detoxification of superoxide free radicals generated in many human organs. In previous studies, SOD2 overexpression has been found in cancers of the bladder, breast, gastric tract, and prostate, and in acute myeloid leukemia, and is associated 

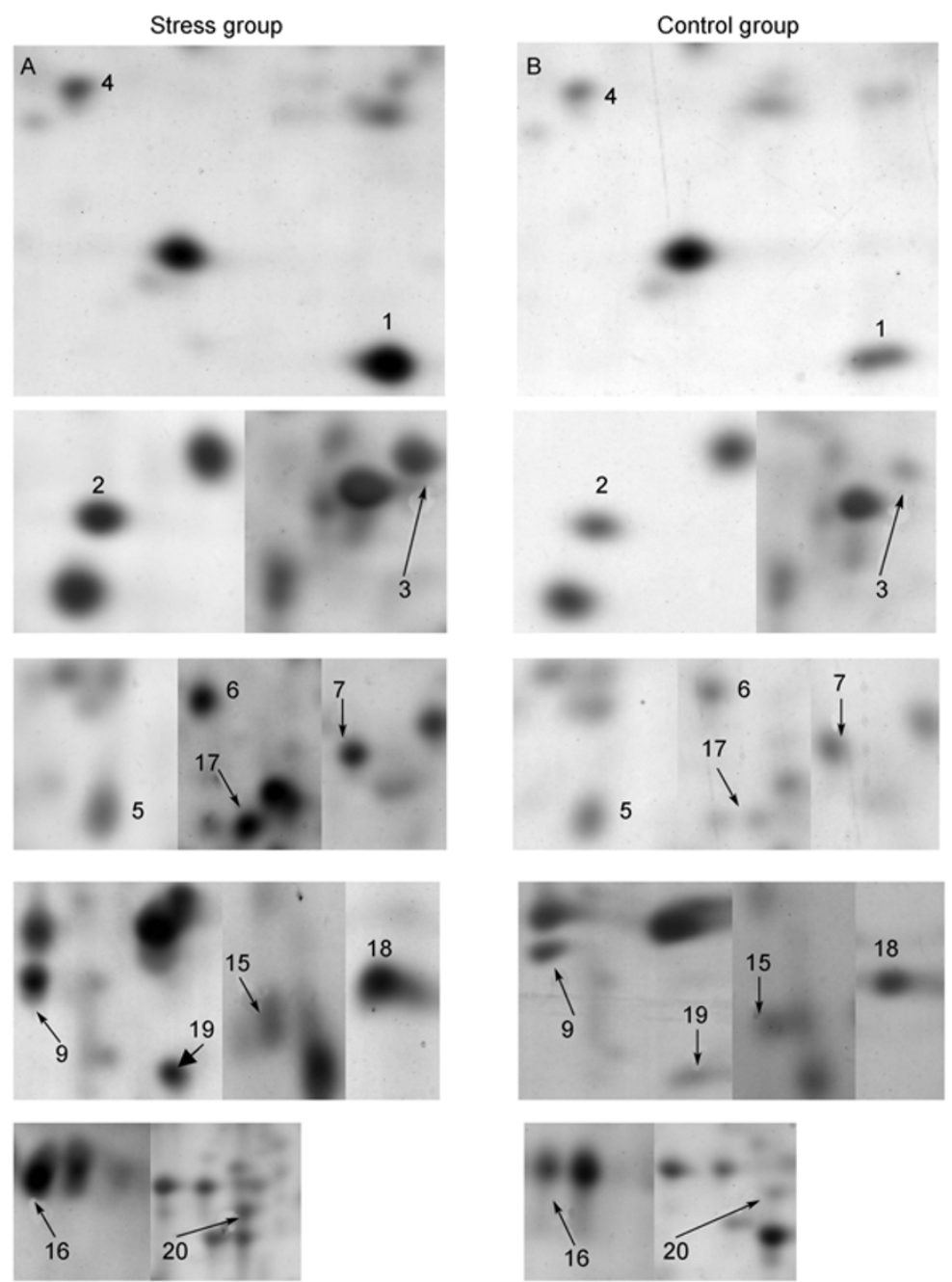

Figure 2 Magnified comparison maps of selected protein spots showing up-regulated protein expression in the stress group compared with the control group. Spots 1, 2, 3, 4, 5, 6, 7, 9, 15, 16, 17, 18, 19 and 20 were significantly up-regulated (average spot intensity increase higher than two-fold compared with the controls) in the psychological stress group (A) at corresponding locations.
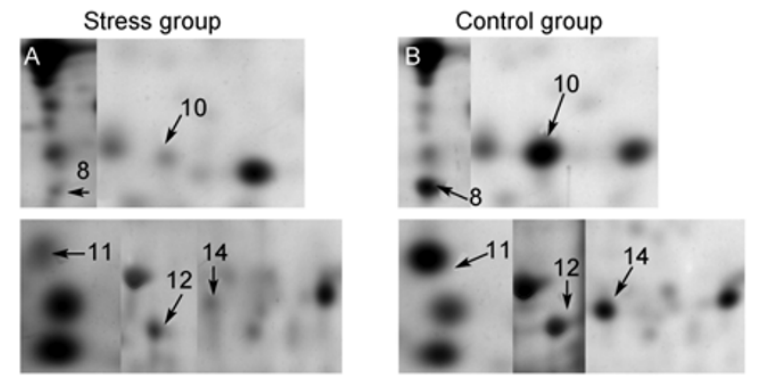

Figure 3 Magnified comparison maps of selected protein spots showing down-regulated protein expression in the stress group compared with the control group. Spots $8,10,11,12$ and 14 were significantly down-regulated (average spot intensity decrease more than two-fold compared with the controls) in the psychological stress group (A) at corresponding locations.

with increased risk for prostate, lung, colorectal, and ovarian cancer [27]. Hirose et al. [28] have shown that increasing SOD1 level promotes tumor cell survival against anti-cancer strategies such as drug therapy and radiation.
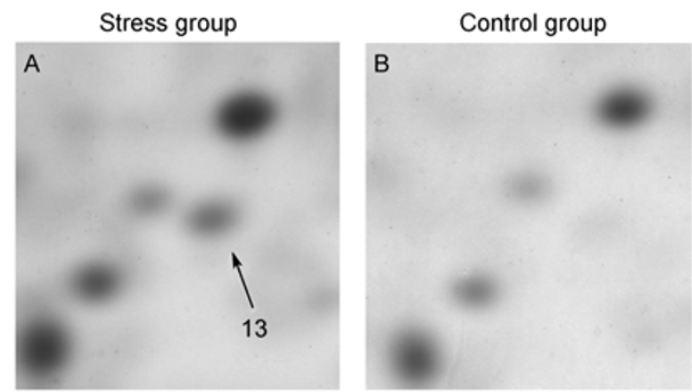

Figure 4 Spot 13 has high expression in the stress group (A), but is absent in the control group (B).

Moreover, Brown et al. [29] reported SOD1 was a key factor in acquired cisplatin resistance in ovarian cancer. Hence, modulation of its activity can lead to sensitization of cisplatin-resistant cells, while using an inhibitor of SOD1 has been found to attenuate angiogenesis and tumor cell proliferation in vitro. Therefore, we speculate that chronic psy- 
Table 1 Xenograft proteins identified by nanoUPLC-ESI-MS/MS to be changed significantly between stress and control groups

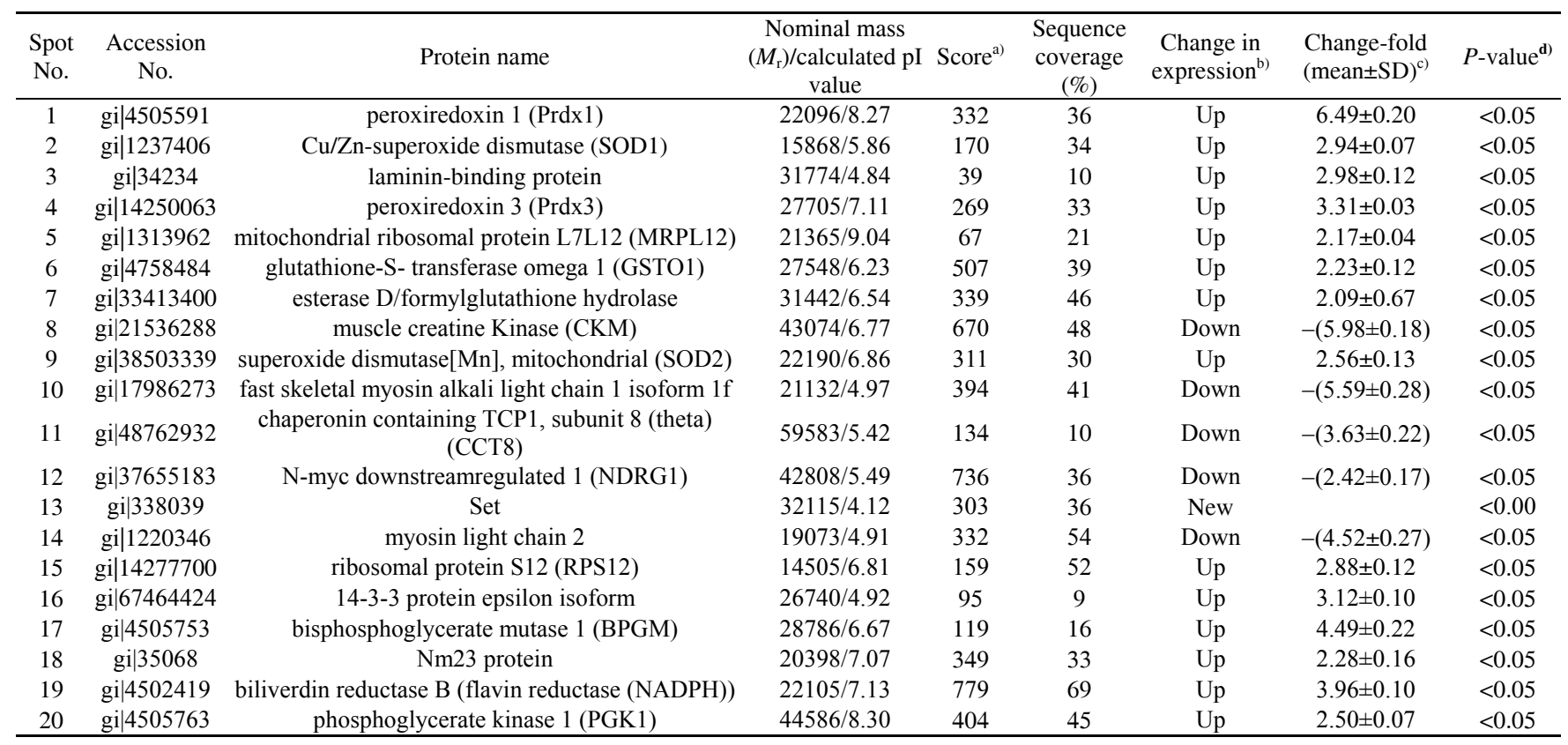

a) Individual ions scores $>38$ indicate identity or extensive homology $(P<0.05)$. b) Up- or down-regulated in the stress group vs. the control group. c) Only the fold changes $\geqslant$ two-fold are shown. Fold changes for down-regulation $\geqslant$ two-fold are denoted by "_". d) Data were analyzed with an independent Student's $t$-test with SPSS version 17.0 software, differences were considered significant if $P<0.05$.

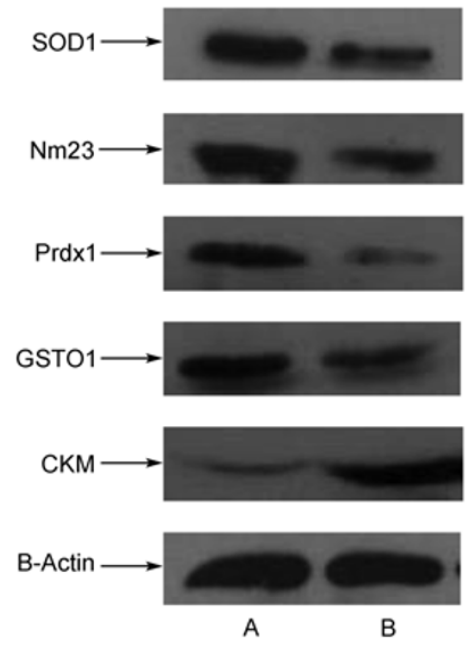

Figure 5 Verification of proteins by Western blot analysis. Lane A, stress group; Lane B, control group.

chological stress can also affect DNA repair, drug transport of the tumor cell and angiogenic processes.

BPGM is a homodimer of a subunit that possesses significant homology with phosphoglycerate mutase (PGM) subunits. Both PGM and PGK1 are involved in the glycolytic cycle and energy metabolism via catalysis reactions in the glycolytic pathway. PGM activity levels are significantly higher in breast tumor than in corresponding normal tissues [30]. Hwang et al. [31] have shown that PGK1 was overexpressed in pancreatic ductal adenocarcinoma, while Tang et al. [32] have reported that overexpression of PGK-1 in lung cancer cells reduces COX-2 expression and pro- motes Th1 immune function. This affects cell invasion, angiogenesis, and immune functions, and ultimately inhibits tumor progression. In the present study, we have observed that it is up-regulated in ovarian cancer tumor following restraint stress, suggesting that this protein may be produced as part of an anti-tumor protective mechanism. This hypothesis requires further exploration.

Ribosomes, the organelles that catalyze protein synthesis, consist of a small 40S subunit and a large 60S subunit. Together these subunits are composed of four RNA species and approximately 80 structurally distinct proteins. Increased expression of RPS12 in colorectal cancers compared with matched normal colonic mucosa has been observed, suggesting this event may be an early event in neoplastic progression to malignancy [33]. Marty et al. [34] have shown that mRNA for MRPL12 (a translational regulator of mitochondrial mRNAs) was highly expressed in the colon, in which a reduction in mitochondrial activity (as may be expected following disruption of mitochondrial protein production) is associated with tumor formation. Studies on the relationship between ribosomal proteins and ovarian cancer deserve to be further explored.

nm23 gene is a reported metastasis suppressor gene, but the role of $n m 23$ in human ovarian cancer is still controversial. Younl et al. [35] and Schneider et al. [36] have shown that $\mathrm{nm} 23$ overexpression was an independent factor associated with a significantly worse early stage prognosis and decreased overall survival in patients with ovarian carcinoma. Thus, nm23 may have a biological function that leads to poor clinical outcomes in ovarian carcinoma. It is unclear whether, in the current study, up-regulation of nm23 in the 
stress group is related to a worse prognosis.

14-3-3 proteins are a family of dimeric proteins involved in many pathways of signal transduction. Seven isoforms have been described and various isoforms carry out important functions in apoptotic control. 14-3-3 protein epsilon, as an anti-apoptotic protein, is up-regulated in tumor following stress exposure. Its function is to activate tyrosine and tryptophan hydroxylases in the presence of $\mathrm{Ca}^{2+} /$ calmodulin-dependent protein kinase [37]. However, it is probably a multifunctional regulator of the cell signaling processes mediated by both kinases.

Other proteins that are overexpressed in the stress group are involved in the regulation of tumor cell proliferation and differentiation. Laminin-binding protein is well-correlated with the biological aggressiveness of cancer cells [38]. GSTO1 acts as a small stress response protein, which is likely involved in cellular redox homeostasis, which controls the conjugation of reduced glutathione to a wide number of exogenous and endogenous hydrophobic electrophiles [37]. Esterase D may be involved in the recycling of sialic acids, and is used as a genetic marker for retinoblastoma and Wilson's disease [39]. The final step in heme metabolism in mammals is catalyzed by NADPH, which is considered to be related to drug resistance and anti-apoptosis, and plays a role in regulating cellular redox homeostasis and signaling pathways [40].

Myosin is a hexameric ATPase cellular motor protein. As an important member of the cytoskeleton components, the myosin family is involved in a number of physiological activities, such as morphology maintenance and material transportation. This proteomic study has observed significant down-regulation of myosin in xenograft tissues after stress treatment suggesting that psychological stress effects might be associated with cell physiological activities, such as destruction of the cytoskeletal system. Zhou et al. [41] have shown that reduction of myosin light-chain kinase in breast cancer cells was able to suppress cell proliferation and migration. Ren et al. [42] have suggested that overexpression of myofibrillogenesis regulator 1 is associated with cancer cell proliferation and migration mediated by myosin light chain 2 . These results of previous research are in apparent contradiction, suggesting the need for further research to verify the role of myosin and other cytoskeletal proteins in tumor progression.

Creatine kinase $(\mathrm{CK})$ is a ubiquitous enzyme that catalyses the reversible transphosphorylation reaction between ATP and creatine, which plays a key role in the energy metabolism of cells with high and fluctuating energy demands. Previous studies have shown that CK activity in neoplastic tissue was lower than that of normal tissue when the activity is reported as a function of the extracted protein [30]. CCT8 is a molecular chaperone, and plays an important role in the assembly of actin and tubulin, which is significant given that a reduction in the number of microtubules is an important feature of malignant transformation in cells. Myung et al. [43] reported that chaperones were expressed in 10 human tumor cell lines. Both $\mathrm{CK}$ and CCT8 are down-regulated in the stress group, which would give further support to the hypothesis that psychological stress affects tumor cell energy metabolism and the cytoskeletal system.

NDRG1 was found to be down-regulated in the xenografts of the stress group compared with the control group. NDRG1 has been reported to be necessary for p53-mediated apoptosis and it plays a role in suppressing malignant cell growth. NDRG1 expression is significantly lower in patients with lymph node and bone metastasis, and thus NDRG1 is thought to function as a metastasis suppressor in several cancers, such as colon cancer and esophageal squamous cell carcinoma $[44,45]$.

set is a putative oncogene associated with myeloid leukemogenesis, and SET protein has been reported as an inhibitor of tumor suppressor PP2A in chronic myeloid leukemia [46]. Ouellet et al. [47] have shown that SET complexes were highly expressed in invasive grade 3 serous epithelial ovarian cancer. In this study, we found that SET protein was only expressed in the stress group. However, little is known about this protein and its actions, and further study is required before inferring anything about its role in cancer progression.

The present study has indicated that exposure to chronic psychological stress might lead to significant changes in the proteomic profile of tumors. Expression alterations of those proteins might affect tumor cell growth by regulating energy metabolism, cytoskeletal organization, and cellular proliferation, differentiation, and apoptosis. Therefore, the present results provide new evidence that could be helpful in further understanding the molecular relationship between psychological stress and tumor progression.

This work was supported by the National Natural Science Foundation of China (Grant Nos. 30860301 and 30660192).

1 Lynch H T, Casey M J, Lynch J, et al. Genetics and ovarian carcinoma. Semin Oncol, 1998, 25: 265-280

2 Li Z, Zhao X, Yang J, et al. Proteomics profile changes in cisplatin-treated human ovarian cancer cell strain. Sci China Ser C-Life Sci, 2005, 48: 648-657

3 Kiecolt-Glaser J K, Robles T F, Heffner K L, et al. Psycho-oncology and cancer: psychoneuroimmunology and cancer. Ann Oncol, 2002, 13: $165-169$

4 Antoni M H. Psychoneuroendocrinology and psychoneuroimmunology of cancer: plausible mechanisms worth pursuing. Brain Behav Immun, 2003, 17: 84-91

5 Boyce E A, Kohn E C. Ovarian cancer in the proteomics era: diagnosis, prognosis, and therapeutics targets. Int J Gynecol Cancer, 2005, 15: 266-273

6 Philip R, Murthy S, Krakover J, et al. Shared Immuno-proteome for ovarian cancer diagnostics and immunotherapy: potential theranostic approach to cancer. J Proteome Res, 2007, 6: 2509-2517

7 Gortzak-Uzan L, Ignatchenko A, Evangelou A I, et al. A proteome resource of ovarian cancer ascites: integrated proteomic and bioinformatic analyses to identify putative biomarkers. J Proteome Res, 2008, 7: 339-351

8 Zhu Y, Wu R, Sangha N, et al. Classifications of ovarian cancer tis- 
sues by proteomic patterns. Proteomics, 2006, 6: 5846-5856

9 Wang J, Zhang X, Ge X, et al. Proteomic studies of early-stage and advanced ovarian cancer patients. Gynecol Oncol, 2008, 111: 111-119

10 Young T, Mei F, Liu J, et al. Proteomics analysis of H-RAS-mediated oncogenic transformation in a genetically defined human ovarian cancer model. Oncogene, 2005, 24: 6174-6184

11 Jackson D, Craven R A, Hutson R C, et al. Proteomic profiling identifies afamin as a potential biomarkerfor ovarian cancer. Clin Cancer Res, 2007, 13: 7370-7379

12 Davidson B, Espina V, Steinberg S M, et al. Proteomic analysis of malignant ovarian cancer effusions as a tool for biologic and prognostic profiling. Clin Cancer Res, 2006, 12: 791-799

13 Ye B, Cramer D W, Skates S J, et al. Haptoglobin- $\alpha$ subunit as potential serum biomarker in ovarian cancer: identification and characterization using proteomic profiling and mass spectrometry. Clin Cancer Res, 2003, 9: 2904-2911

14 Uys J D, Hattingh S M, Stein D J, et al. Large scale hippocampal cellular distress may explain the behavioral consequences of repetitive traumatic experiences-a proteomic approach. Neurochem Res, 2008, 33: 1724-1734

15 Teague C R, Dhabhar F S, Barton R H, et al. Metabonomic studies on the physiological effects of acute and chronic psychological stress in Sprague-Dawley rats. J Proteome Res, 2007, 6: 2080-2093

16 Carboni L, Piubelli C, Pozzato C, et al. Proteomic analysis of rat hippocampus after repeated psychological stress. Neuroscience, 2006, 137: 1237-1246

17 Masters J R, Thomson J A, Daly-Burns B, et al. Short tandem repeat profiling provides an international reference standard for cell lines. Proc Natl Acad Sci USA, 2001, 98: 8012-8017

18 Park S J, Kim M J, Kim H B, et al. Trichostatin A sensitizes human ovarian cancer cells to TRAIL-induced apoptosis by down-regulation of c-FLIPL via inhibition of EGFR pathway. Biochem Pharmacol, 2009, 77: 1328-1336

19 Stangelberger A, Schally A V, Varga J L, et al. Inhibitory effect of antagonists of bombesin and growth hormone-releasing hormone on orthotopic and intraosseous growth and invasiveness of PC-3 human prostate cancer in nude mice. Clin Cancer Res, 2005, 11: 49-57

20 Alfonso J, Frick L R, Silberman D M, et al. Regulation of hippocampal gene expression is conserved in two species subjected to different stressors and antidepressant treatments. Biol Psychiatry, 2006, 59: 244-251

21 Sheridan J F, Dobbs C, Jung J, et al. Stress-induced neuroendocrine modulation of viral pathogenesis and immunity. Ann NY Acad Sci, 1998, 840: 803-808

22 Kim S, Park Y W, Schiff B A, et al. An orthotopic model of anaplastic thyroid carcinoma in athymic nude mice. Clin Cancer Res, 2005, 11: $1713-1721$

23 Winter D, Kugelstadt D, Seidler J, et al. Protein phosphorylation influences proteolytic cleavage and kinase substrate properties exemplified by analysis of in vitro phosphorylated Plasmodium falciparum glideosome-associated protein 45 by nano-ultra performance liquidchromatography-tandem mass spectrometry. Anal Biochem, 2009, 393: 41-47

24 Culp W D, Neal R, Massey R, et al. Proteomic analysis of tumor establishment and growth in the B16-F10 mouse melanoma model. J Proteome Res, 2006, 5: 1332-1343

25 Nonn L, Berggren M, Powis G. Increased expression of mitochondrial peroxiredoxin-3 (thioredoxin peroxidase-2) protects cancer cells against hypoxia and drug-induced hydrogen peroxide-dependent apoptosis. Mol Cancer Res, 2003, 1: 682-689

26 Kim J H, Bogner P N, Baek S H, et al. Up-regulation of peroxiredoxin1 in lung cancer and its implication as a prognostic and therapeutic target. Clin Cancer Res, 2008, 14: 2326-2333

27 Kang D, Lee K M, Park S K, et al. Functional variant of manganese superoxide dismutase (SOD2 V16A) polymorphism is associated with prostate cancer risk in the prostate, lung, colorectal, and ovarian cancer study. Cancer Epidemiol Biomarkers Prev, 2007, 16: 1581-1586
28 Hirose K, Longo D L, Oppenheim J J, et al. Overexpression of mitochondrial manganese superoxide dismutase promotes the survival of tumor cells exposed to interleukin-1, tumor necrosis factor, selected anticancer drugs, and ionizing radiation. FASEB J, 1993, 7: 361-368

29 Brown D P, Chin-Sinex H, Nie B, et al. Targeting superoxide dismutase 1 to overcome cisplatin resistance in human ovarian cancer. Cancer Chemother Pharmacol, 2009, 63: 723-730

30 Durany N, Joseph J, Jimenez O M, et al. Phosphoglycerate mutase, 2,3-bisphosphoglycerate phosphatase, creatine kinase and enolase activity and isoenzymes in breast carcinoma. Br J Cancer, 2000, 82: 20-27

31 Hwang T L, Liang Y, Chien K Y, et al. Overexpression and elevated serum levels of phosphoglycerate kinase 1 in pancreatic ductal adenocarcinoma. Proteomics, 2006, 6: 2259-2272

32 Tang S J, Ho M Y, Cho $\mathrm{H} \mathrm{C}$, et al. Phosphoglycerate kinase 1-overexpressing lung cancer cells reduce cyclooxygenase 2 expression and promote anti-tumor immunity in vivo. Int J Cancer, 2008, 123: 2840-2848

33 Pogue-Geile K, Geiser J R, Shu M, et al. Ribosomal protein genes are overexpressed in colorectal cancer: isolation of a cDNA clone encoding the human S3 ribosomal protein. Mol Cell Biol, 1991, 11: 3842-3849

34 Marty L, Taviaux S, Fort P. Expression and human chromosomal localization to $17 \mathrm{q} 25$ of the growth-regulated gene encoding the mitochondrial ribosomal protein MRPL12. Genomics, 1997, 41: 453-457

35 Youn B S, Kim D S, Kim J W, et al. NM23 as a prognostic biomarker in ovarian serous carcinoma. Mod Pathol, 2008, 21: 885-892

36 Schneider J, Pollán M, Jiménez E, et al. nm23-H1 expression defines a high-risk subpopulation of patients with early-stage epithelial ovarian carcinoma. Br J Cancer, 2000, 82: 1662-1670

37 Castagna A, Antonioli $\mathrm{P}$, Astner $\mathrm{H}$, et al. A proteomic approach to cisplatin resistance in the cervix squamous cell carcinoma cell line A431. Proteomics, 2004, 4: 3246-3267

38 Satoh K, Narumi K, Abe T, et al. Diminution of 37-kDa laminin binding protein expression reduces tumour formation of murine lung cancer cells. Br J Cancer, 1999, 80: 1115-1122

39 Young L J, Lee E Y, To H A, et al. Human esterase D gene: complete cDNA sequence, genomic structure and application in the genetic diagnosis of human retinoblastoma. Hum Genet, 1988, 79: 137-141

40 Xia C, Meng Q, Liu L Z, et al. Reactive oxygen species regulate angiogenesis and tumor growth through vascular endothelial growth factor. Cancer Res, 2007, 67: 10823-10830

41 Zhou X, Liu Y, You J, et al. Myosin light-chain kinase contributes to the proliferation and migration of breast cancer cells through cross-talk with activated ERK1/2. Cancer Lett, 2008, 270: 312-327

42 Ren K, Jin H, Bian C, et al. MR-1 modulates proliferation and migration of human hepatoma HepG2 cells through myosin light chains-2 (MLC2)/focal adhesion kinase (FAK)/Akt signaling pathway. J Biol Chem, 2008, 283: 35598-35605

43 Myung J K, Afjehi-Sadat L, Felizardo-Cabatic M, et al. Expressional patterns of chaperones in ten human tumor cell lines. Proteome Sci, 2004, 2: 8-13

44 Ando T, Ishiguro H, Kimura M, et al. Decreased expression of NDRG1 is correlated with tumor progression and poor prognosis in patients with esophageal squamous cell carcinoma. Dis Esophagus, 2006, 19: 454-458

45 Ellen T P, Ke Q, Zhang P, et al. NDRG1, a growth and cancer related gene: regulation of gene expression and function in normal and disease states. Carcinogenesis, 2008, 29: 2-8

46 Neviani P, Santhanam R, Trotta R, et al. The tumor suppressor PP2A is functionally inactivated in blast crisis CML through the inhibitory activity of the BCR/ABL-regulated SET protein. Cancer Cell, 2005, 8: 355-368

47 Ouellet V, Le Page C, Guyot M C, et al. SET complex in serous epithelial ovarian cancer. Int J Cancer, 2006, 119: 2119-2126

Open Access This article is distributed under the terms of the Creative Commons Attribution License which permits any use, distribution, and reproduction in any medium, provided the original author(s) and source are credited. 